\title{
Multivariable Fuzzy Control for Prosthetic Hands
}

\author{
Xiao-Gang Duan ${ }^{1,}$, , Huan-Xin Luo ${ }^{1}$, Hua Deng ${ }^{1}$ \\ ${ }^{1}$ School of Mechanical \& Electrical Engineering, Central South University, Changsha 410083, \\ China \\ aemail: xgduan@csu.edu.cn
}

Keywords: Multi-variables, fuzzy logic control, prosthetic hand

Abstract. Multi-freedom prosthetic hand is multivariable system which has many inputs and outputs. The design of fuzzy logic controller is very complex because so many fuzzy control systems have to be designed based on conventional idea. Thus, a multivariable fuzzy control is proposed. An effective strategy is that all variables share a common membership function and a rule base. It reduces complexity of membership function and rule base. Finally, the proposed multivariable Fuzzy logic controller is used to control a multi-freedom prosthetic hand. The control performance shows that the multivariable is effective.

\section{Introduction}

Multi-freedom prosthetic hand is a multivariable system which has many inputs and outputs. Among various control methods for multivariable systems, proportional integral derivative (PID) controllers are still popularly used in industry. Although there has many PID design methods, tuning of its gains is always challenging for optimum operations. Generally speaking, PID controller may not perform well because practical systems are often complex, nonlinear, high-order, etc. Moreover, there is multivariable coupling and environmental disturbance. Because the PID controller is simple, the overall control performance with respect to transient and steady state operations is always poorer compared with other advanced techniques such as model predictive control, sliding model control, fuzzy control, etc.. However, it has been found that fuzzy-logic-based PID controllers have better capabilities of handling the aforesaid systems [1]-[2].

The difficulty in using fuzzy logic controller is to properly design membership function (MF) and rule base. The most popular approach used before is to select them based on the expert's experience. A linguistic variable can mean different things to different people [3], resulting in different interpretation for MFs and rules, and thus different control performance may be produced [4]. Many researchers have made contributions to multivariable fuzzy controls. These fuzzy controls can be classified as two methods, composition and decomposition of multivariable fuzzy system [5-6]. Since the former is very complex, it is nor proper for industrial processes. Thus, many investigations have been made with the aim of reducing this complexity by decomposing the fuzzy control rule bases [5-6]. The idea of the decomposing fuzzy system is to decouple multivariable fuzzy system into a set of lower dimensional systems. A classical application of multivariable fuzzy systems is to design fuzzy PID controller, where three parameters of PID controller are tuned by three fuzzy systems respectively. Although the decomposition of multivariable control rules alleviates the complexity of the problem, the workload is still larger because so many fuzzy control systems have to be designed. A feasible method is that all variables share a common membership function and a rule base. Although a few researchers have used this common rule base to design 
fuzzy controller preliminarily [7-8], the theory is still lack.

Motivated by above discussions, a multivariable fuzzy control strategy is proposed for multi-freedom prosthetic hands in this paper. A key strategy is that all variables share a common membership function and a rule base. It reduces complexity of membership function and rule base. Finally, the proposed multivariable Fuzzy logic controller is used to control a multi-freedom prosthetic hand. The control performance shows that the multivariable is effective.

\section{Problem Formulation}

A five-finger robotic hand [9] can be described by the following dynamic model

$$
I(\mathbf{x})+C(\mathbf{x},+\mathbf{x})=\boldsymbol{\tau}
$$

where $\mathbf{x}=\left[x_{1}, \mathrm{~L}, x_{N}\right]^{T}$ is the state vector, $\boldsymbol{\tau}=\left[\tau_{1}, \mathrm{~L}, \tau_{M}\right]^{T}$ is the input vector, $I(\mathbf{x})$ is the inertia matrix, $C(\mathbf{x}, \mathbf{A})$ is the Coriolis vector, $G(\mathbf{x})$ is the gravity or elastic vector.

If the system in (1) can be decentralized into $N(N=M)$ single input and single output (SISO) subsystems, the design of multivariable fuzzy controller is reduced as $N$ fuzzy subsystem [5], as shown in Fig. 1.

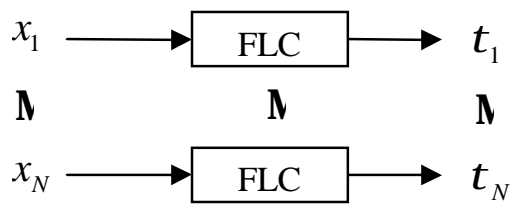

(b)

Fig. 1. Multivariable systems by decentralized fuzzy control

The complexity is summarized as follows

(1) Generally, every variable needs to design a membership function. Thus, the design complexity is increased as the number of variable is increasing.

(2) Rule base is very complex. The rule base design is also complex because each variable needs a rule base.

Thus, a multivariable fuzzy logic control is presented in the following of the paper.

\section{Multivariable FLC}

The multivariable FLC has the similar basic structure with a traditional one, as shown in Fig. 2, where $\mathbf{e}=\left[e_{1}, \mathrm{~L}, e_{n}\right]^{T}$ and $\&\left[\&_{\mathbb{L}} \mathrm{L}, \bigotimes_{n}\right]^{T}$ are two crisp input vectors, and $\mathbf{u}=\left[u_{1}, \mathrm{~L}, u_{n}\right]^{T}$ is crisp output vector. The input of the FLC $\&$ is changed as

$$
\boldsymbol{\varepsilon}=H(\cdot) \mathbf{e}, \quad \mathbf{v}=W(\cdot) \boldsymbol{\varepsilon}
$$

with 


$$
H(\cdot)=\left[\begin{array}{ccc}
h_{1}(\cdot) & 0 & 0 \\
0 & 0 & 0 \\
0 & 0 & h_{n}(\cdot)
\end{array}\right], W(\cdot)=\left[\begin{array}{ccc}
w_{1}(\cdot) & 0 & 0 \\
0 & 0 & 0 \\
0 & 0 & w_{n}(\cdot)
\end{array}\right]
$$

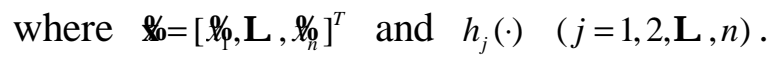

This multivariable FLC is also composed of fuzzifier, rules, inference, and defuzzifier. The difference is that the proposed FLC can process multi-variables.

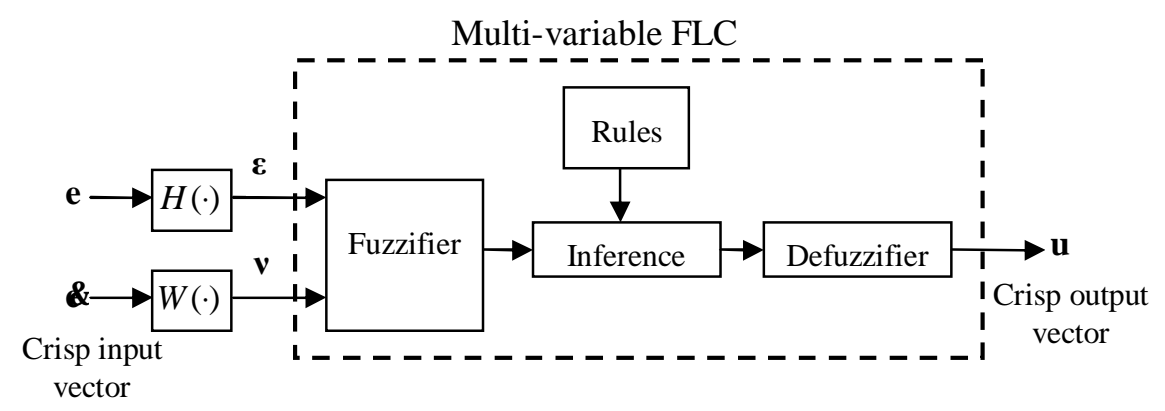

Fig. 2. The structure of Multi-variable FLC

\section{Fuzzifier}

The fuzzifier maps crisp input vector $\boldsymbol{\varepsilon}, \boldsymbol{v} \in R^{n}$ into fuzzy set $A_{I}=\left[A_{\varepsilon}, A_{\downarrow}\right]$, where $A_{\varepsilon}$ and $A_{v}$ are fuzzy sets of input vector $\boldsymbol{\varepsilon}$ and $\mathbf{v}$, respectively. The most widely used fuzzifier is the singleton fuzzifier. Thus, the singleton fuzzifier is adopted. $A_{\varepsilon}$ is fuzzy singleton with support $\varepsilon^{\prime}$ if $\boldsymbol{\mu}_{A_{\varepsilon}}(\boldsymbol{\varepsilon})=\left[1_{1}, \mathrm{~L}, 1_{n}\right]^{T}$ for $\boldsymbol{\varepsilon}=\boldsymbol{\varepsilon}^{\prime}$ and $\boldsymbol{\mu}_{A_{\varepsilon}}(\boldsymbol{\varepsilon})=\left[0_{1}, \mathrm{~L}, 0_{n}\right]^{T}$ for $\boldsymbol{\varepsilon} \neq \boldsymbol{\varepsilon}^{\prime} . \quad A$ is fuzzy singleton with support $\mathbf{v}^{\prime}$ if $\boldsymbol{\mu}_{A_{1}}(\boldsymbol{v})=\left[1_{1}, \mathrm{~L}, 1_{n}\right]^{T}$ for $\mathbf{v}=\mathbf{v}^{\prime}$ and $\boldsymbol{\mu}_{A_{v}}(\boldsymbol{v})=\left[0_{1}, \mathrm{~L}, 0_{n}\right]^{T}$ for $\mathbf{v} \neq \mathbf{v}^{\prime}$.

\section{Rules}

In order to simplify rule base, a rule base is used. The difference between the proposed and traditional rule base is that each fuzzy input is a vector in the former and a single variable in the later. Thus, the rules have multi-variables property. The $l$-th rule is given as

$$
S^{l}: \text { IF } \boldsymbol{\varepsilon} \text { is } C^{l} \text { and } \mathbf{v} \text { is } D^{l} \text {, THEN } \mathbf{u} \text { is } G^{l} .
$$

This rule represents a fuzzy relation between the input space and the output space.

\section{Inference Engine}

In the inference engine, fuzzy logic principles are used to combine fuzzy IF-THEN rules from the fuzzy rule base into a mapping from fuzzy input sets to fuzzy output sets. Based on the $l$-th rule (14), a multi-variable fuzzy relation is given as follows

$$
S^{l}: C^{l} \times D^{l} \rightarrow G^{l} \quad l=1,2, \mathrm{~L}, M
$$

where $M$ denotes the number of rules. 
Fuzzy set $A_{I}$ firing the rule $S^{l}$ is denoted by

$$
A_{I} \mathrm{OS} S^{l}=\left[A_{\varepsilon}, A_{V}\right] \mathrm{O}\left(C^{l} \times D^{l} \rightarrow G^{l}\right)
$$

Each rule $R^{l}$ determines a fuzzy set $B^{l}=A_{I}$ o $R^{l}$ such that

$$
\begin{aligned}
& \boldsymbol{\mu}_{B^{\prime}}(\mathbf{u})=\boldsymbol{\mu}_{A_{1} \mathrm{os}^{l}}(\mathbf{u}) \\
& =\sup _{\boldsymbol{\varepsilon}, v \in R^{n}}\left\{\left[\boldsymbol{\mu}_{A_{\varepsilon}}(\boldsymbol{\varepsilon}) * \boldsymbol{\mu}_{A_{v}}(\mathbf{v})\right] *\left[\boldsymbol{\mu}_{C^{l}}(\boldsymbol{\varepsilon}) * \boldsymbol{\mu}_{D^{l}}(\mathbf{v})\right] * \boldsymbol{\mu}_{G^{l}}(\mathbf{u})\right\} \\
& =\boldsymbol{\mu}_{G^{\prime}}(\mathbf{u}) *\left\{\left[\sup _{\boldsymbol{\varepsilon}, \boldsymbol{v} \in R^{n}} \boldsymbol{\mu}_{A_{\varepsilon}}(\boldsymbol{\varepsilon}) * \boldsymbol{\mu}_{C^{l}}(\boldsymbol{\varepsilon})\right] *\left[\sup _{\boldsymbol{\varepsilon}, v \in R^{n}} \boldsymbol{\mu}_{A^{\prime}}(\mathbf{v}) * \boldsymbol{\mu}_{D^{\prime}}(\mathbf{v})\right]\right\}
\end{aligned}
$$

where $\boldsymbol{\mu}_{B^{\prime}}(\mathbf{u})=\left[\mu_{B_{1}^{\prime}}\left(u_{1}\right), \mathrm{L}, \mu_{B_{n}^{\prime}}\left(u_{n}\right)\right]^{T}$, and

$$
\begin{aligned}
& \boldsymbol{\mu}_{A_{\varepsilon}}(\boldsymbol{\varepsilon}) * \boldsymbol{\mu}_{C^{l}}(\boldsymbol{\varepsilon})=\left[\begin{array}{llll}
\boldsymbol{\mu}_{A_{\varepsilon}}\left(\varepsilon_{1}\right) * \boldsymbol{\mu}_{C^{l}}\left(\varepsilon_{1}\right) & \mathrm{L} & \boldsymbol{\mu}_{A_{\varepsilon}}\left(\varepsilon_{n}\right) * \boldsymbol{\mu}_{C^{l}}\left(\varepsilon_{n}\right)
\end{array}\right]^{T} \\
& \boldsymbol{\mu}_{A_{1}}(v) * \boldsymbol{\mu}_{D^{\prime}}(\mathbf{v})=\left[\begin{array}{llll}
\boldsymbol{\mu}_{A_{1}}\left(v_{1}\right) * \boldsymbol{\mu}_{D^{l}}\left(v_{1}\right) & \mathrm{L} & \boldsymbol{\mu}_{A_{1}}\left(v_{n}\right) * \boldsymbol{\mu}_{D^{\prime}}\left(v_{n}\right)
\end{array}\right]^{T}
\end{aligned}
$$

Combining all fired rules, one can obtains

$$
B=\bigcup_{l=1}^{M} B^{l}=\bigcup_{l=1}^{M} B^{l}
$$

\section{Defuzzifer}

Defuzzification produces a crisp output vector from all fired rules. Many defuzzifiers have been proposed in the literature, such as maximum, centerof- sums, center-of-sets, etc. [28]. Here, the center-of-sets is chosen in this paper due to its simple computation. Multi crisp outputs can be simplified as follows:

$$
\begin{aligned}
& \mathbf{u}=\sum_{l=1}^{M} Q^{l} \boldsymbol{\mu}_{B^{\prime}} / \cdot \sum_{l=1}^{M} \boldsymbol{\mu}_{B^{l}} \\
& =\left[\begin{array}{lll}
\frac{\sum_{l=1}^{M} q_{1}^{l} \mu_{B_{1}^{l}}}{\sum_{l=1}^{M} \mu_{B_{1}^{l}}} & \mathrm{~L} & \frac{\sum_{l=1}^{M} q_{n}^{l} \mu_{B_{n}^{l}}}{\sum_{l=1}^{M} \mu_{B_{n}^{l}}}
\end{array}\right]^{T}
\end{aligned}
$$

with

$$
Q^{l}=\operatorname{diag}\left[\begin{array}{lll}
q_{1}^{l} & \mathrm{~L} & q_{n}^{l}
\end{array}\right]
$$

where " $/ . "$ denotes a division operation that is defined as a division of each vector components.

\section{Applied to Control a Multi-finger Prosthetic Hand}

\section{Dynamics of the multi-finger prosthetic hand}

The proposed fuzzy logic control was applied to control an underactuated prosthetic hand with multi freedom, as shown in Fig. 3. The underactuated prosthetic hand has five fingers. Each finger has three knuckles, proximal phalanx, middle phalanx and distal phalanx. Only one actuator is installed on the proximal phalanx finger, as shown in Fig. 4. 


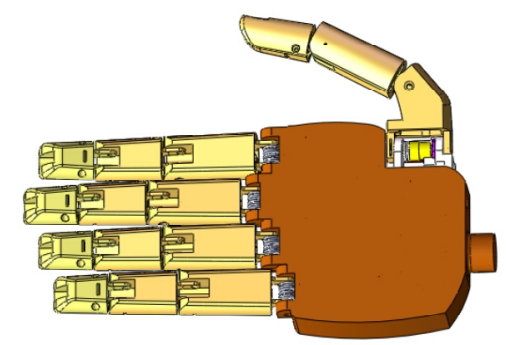

Fig. 3 an underactuated prosthetic hand with multi freedom

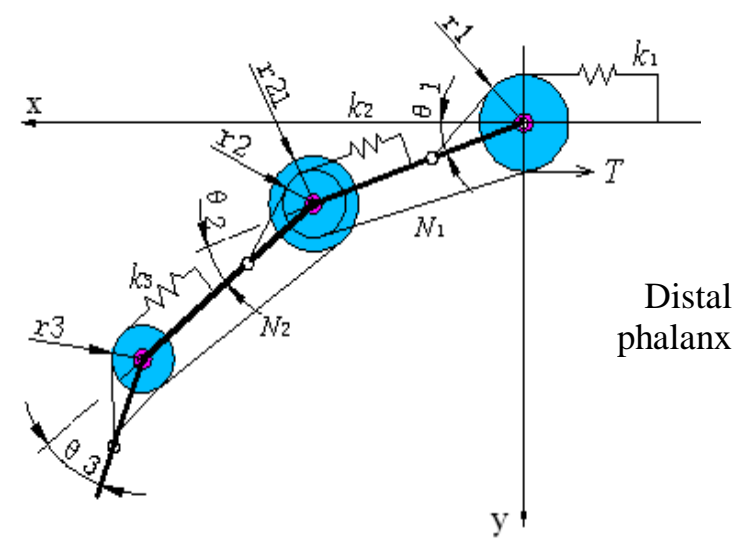

Fig. 4 The scheme of underactuated finger

Generally, each finger needs to be designed a FLC. Five fingers need five FLCs. Thus, the proposed fuzzy logic controller is adopted. For simplicity, only distal phalanx is contacted with the grasped object, as shown in Fig. 4, where $\mathbf{f}$ is the vector of grasping. In the contact force space, the dynamics of the prosthetic hand is described by

$$
M(\mathbf{q}) \boldsymbol{\Phi} \times(\mathbf{q}, \boldsymbol{\phi})+G(\mathbf{q})=\boldsymbol{\tau}-L \mathbf{f}
$$

with

$$
\begin{gathered}
\mathbf{q}=\left[\theta_{11}, \mathrm{~L}, \theta_{i j}, \mathrm{~L}, \theta_{I J}\right]^{T} \\
L=\operatorname{diag}\left(l_{11}, \mathrm{~L}, l_{i j}, \mathrm{~L}, l_{I J}\right) \\
\mathbf{f}=\left[f_{11}, \mathrm{~L}, f_{i j}, \mathrm{~L}, f_{I J}\right]^{T}
\end{gathered}
$$

where $M(\mathbf{q})$ is inertia matrix, $C(\mathbf{q}, \oint)$ is the Coriolis or centripetal vector and $G(\mathbf{q})$ is the elastic vector, $\boldsymbol{\tau}$ is the input torque vector, $\theta_{i, j}$ and $f_{i, j}$ denote the angular displacement and the contact force of the $i$-th finger and the $j$-th dactylus respectively, $i=1, \mathrm{~L}, I$ and $j=1, \mathrm{~L}, J, I$ and $J$ denote the number of fingers that grasps an object and the number of dactylus. Here for the finger in Fig. $3, \quad J=3$.

\section{Design of multivariable FLC}

The multivariable FLC is designed, as shown in Fig. 5. The input $\boldsymbol{\varepsilon}$ and $\mathbf{v}$ as well as the incremental control output $\mathbf{u}^{\prime}$ are classified into seven linguistic labels as positive large (PL), positive middle (PM), positive small (PS), zero (ZR), negative small (NS), negative middle (NM), and negative large (NL). For convenience, the MF is chosen as triangle-shaped MF as indicated in Fig. 6. 


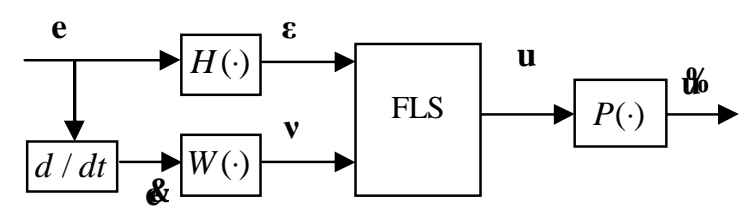

Fig. 5. Multi-variable FLC for multi-freedom prosthetic hand

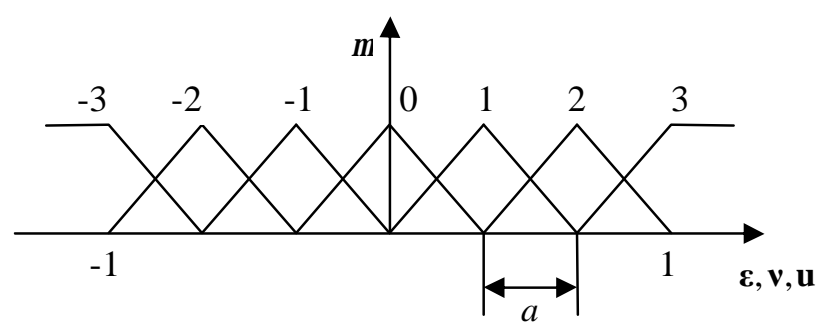

Fig. 6: Membership functions of input and output variable.

The $l$-th rule is defined as

$$
R^{l}: \text { If } \boldsymbol{\varepsilon} \text { is } C^{l} \text { and } \mathbf{v} \text { is } D^{l} \text {, then } \mathbf{u} \text { is } G^{l}
$$

A traditional rule base is used in Table 1.

Table 1. Rule base

\begin{tabular}{cccccccc}
\hline $\boldsymbol{\varepsilon} / \mathbf{v}$ & $\mathrm{NL}$ & $\mathrm{NM}$ & $\mathrm{NS}$ & $\mathrm{ZR}$ & $\mathrm{PS}$ & $\mathrm{PM}$ & $\mathrm{PL}$ \\
\hline $\mathrm{PL}$ & $\mathrm{zr}$ & $\mathrm{ps}$ & $\mathrm{pm}$ & $\mathrm{pl}$ & $\mathrm{pl}$ & $\mathrm{pl}$ & $\mathrm{pl}$ \\
$\mathrm{PM}$ & $\mathrm{ns}$ & $\mathrm{zr}$ & $\mathrm{ps}$ & $\mathrm{pm}$ & $\mathrm{pl}$ & $\mathrm{pl}$ & $\mathrm{pl}$ \\
$\mathrm{PS}$ & $\mathrm{nm}$ & $\mathrm{ns}$ & $\mathrm{zr}$ & $\mathrm{ps}$ & $\mathrm{pm}$ & $\mathrm{pl}$ & $\mathrm{pl}$ \\
$\mathrm{ZR}$ & $\mathrm{nl}$ & $\mathrm{nm}$ & $\mathrm{ns}$ & $\mathrm{zr}$ & $\mathrm{ps}$ & $\mathrm{pm}$ & $\mathrm{pl}$ \\
$\mathrm{NS}$ & $\mathrm{nl}$ & $\mathrm{nl}$ & $\mathrm{nm}$ & $\mathrm{ns}$ & $\mathrm{zr}$ & $\mathrm{ps}$ & $\mathrm{pm}$ \\
$\mathrm{NM}$ & $\mathrm{nl}$ & $\mathrm{nl}$ & $\mathrm{nl}$ & $\mathrm{nm}$ & $\mathrm{ns}$ & $\mathrm{zr}$ & $\mathrm{ps}$ \\
$\mathrm{NL}$ & $\mathrm{nl}$ & $\mathrm{nl}$ & $\mathrm{nl}$ & $\mathrm{nl}$ & $\mathrm{nm}$ & $\mathrm{ns}$ & $\mathrm{zr}$ \\
\hline
\end{tabular}

Inference follows the FLC design in section 3.2. Singleton fuzzifier is used for fuzzification, the minimum is used for the t-norm in the intersection operation, and the center-of-sets is used for defuzzification.

\section{Experiment}

The prosthetic hand is used to grip different objects. The control algorithm is developed in NI Labview. The total computation expense is less than 8 milliseconds. Sampling period is $1 \mathrm{~ms}$ for slip detection. The total control system is $10 \mathrm{~ms}$. Experiment procedures are described as follows: Three fingers grasp tennis stably, as shown in Fig. 7. The grasping forces are as shown in Fig. 8. The experimental results show that the multivariable FLC can control the multi-freedom prosthetic hand stably. 


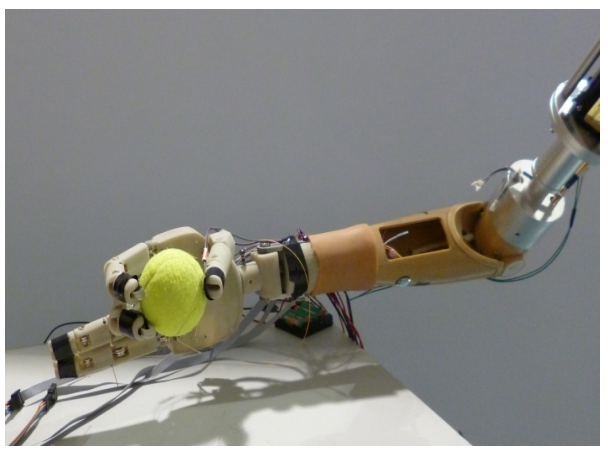

Fig.7. Prosthetic hand grasps tennis stably

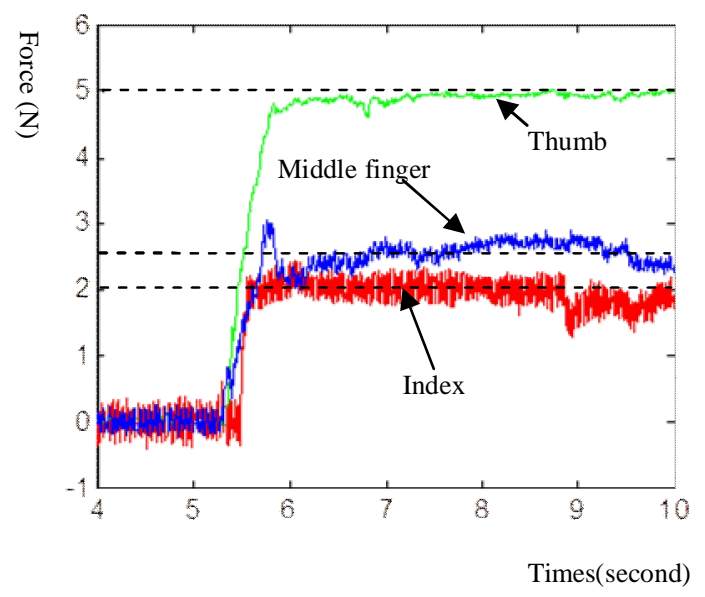

Fig. 8. Grasping force

\section{Conclusion}

A multivariable fuzzy control strategy is presented for multi-freedom prosthetic hands. The structure of the multivariable FLC is constructed. In order to reduce design complexity of the multi-variable FLC, a scaling factor method is used to design membership functions (MFs). Design procedure of multi-variable FLC can be simplified as two steps. (1) Like a traditional FLC, a MF and a rule base are designed. (2) For other MFs, they are transformed to design their scaling factors. It reduces complexity of membership function and rule base. Finally, the proposed multivariable Fuzzy logic controller is used to control a multi-freedom prosthetic hand. The control performance shows that the multivariable is effective.

\section{Acknowledgement}

In this paper, the research was sponsored by the grants from National Basic Research Program 973 of China (Grant No. 2011CB013302).

\section{References}

[1] A. Luo, C. Tang, Z. K. Shuai, J. Tang, X. Y. Xu, and D. Chen, "Fuzzy-PI-based direct-output-voltage control strategy for the STATCOM used in utility distribution systems," IEEE Trans. Ind. Electron., vol. 56, no. 7, pp. 2401-2411, 2009. 
[2] A. Rubaai, and P. Young, "EKF-based PI-/PD-like fuzzy-neural-network controller for brushless drives," IEEE Trans. Ind. Appl., vol. 47, no. 6, pp. 2391-2401, 2011.

[3] J. Jamaludin, N. A. Rahim, and W. P. Hew, "An elevator group control system with a self-tuning fuzzy logic group controller," IEEE Trans. Ind. Electron., vol. 57, no. 12, pp. 4188-4198, 2010.

[4] H.X. Li, L. H. B. Gatland, "A new methodology for designing a fuzzy logic controller," IEEE Trans. Syst. Man, and Cybern., vol. 25, no. 3, pp. 505-512, 1995.

[5] E. Alexander, P. M. Frank, "Decentralised fuzzy control of multivariable systems by passive decomposition," Intelligent Systems Engineering, pp. 194-200, 1994.

[6] P. G. Lee, K. K. Lee, G. J. Jeon, "An index of applicability for the decomposition method of multivariable fuzzy systems," IEEE Transactions on Fuzzy Systems, vol. 3, no. 3, pp. 364-369, 1995.

[7] J. Nie, "Fuzzy control of multivariable nonlinear servomechanisms with explicit decoupling scheme", IEEE Transactions on Fuzzy Systems, vol. 5. no. 2, pp. 304-311, 1997.

[8] E. Harinath, G. K. I. Mann, "Design and tuning of standard additive model based fuzzy PID controllers for multivariable process systems", IEEE Transactions on Systems, Man, and Cybernetics-Part B: Cybernetics, vol. 38, no. 3, pp. 667-674, 2008.

[9] C. H. Chen, D. S. Naidu, "Hybrid control strategies for a five-finger robotic hand", Biomedical Signal Processing and Control, vol. 8, no. 4, pp. 382-390, 2013. 